\title{
Noncoherent Sequence Detection
}

\author{
Giulio Colavolpe, Student Member, IEEE, and Riccardo Raheli, Member, IEEE
}

\begin{abstract}
In this paper, new noncoherent sequence detection algorithms for combined demodulation and decoding of coded linear modulations transmitted over additive white Gaussian noise channels, possibly affected by intersymbol interference, are presented. Optimal sequence detection in the presence of a random rotation of the signal phase, assumed to be constant during the entire transmission, requires a receiver complexity exponentially increasing with the duration of the transmission. Based on proper approximations, simple suboptimal detection schemes based on the Viterbi algorithm are presented, whose performance approaches that of coherent detection. In a companion paper by Colavolpe and Raheli, noncoherent sequence detection is extended to continuous phase modulations.

In the proposed schemes, the tradeoff between complexity and performance is simply controlled by a parameter, referred to as implicit phase memory, and the number of states of a trellis diagram. Besides being realizable, these schemes have the convenient feature of allowing us to remove the constant phase assumption and encompass time-varying phase models. The proposed schemes compare favorably with other solutions previously proposed in the technical literature.
\end{abstract}

Index Terms - Convolutional codes, intersymbol interference, maximum-likelihood detection, noncoherent sequence detection, trellis-coded modulation.

\section{INTRODUCTION}

$\mathbf{T}$ HE problem of optimal detection of a possibly encoded information sequence transmitted over a bandpass additive white Gaussian noise (AWGN) channel is commonly approached by trying to approximately implement coherent detection. In applications in which a coherent phase reference is not available, this approximation is based on the use of a phase-synchronization scheme, that extracts a phase reference from the incoming signal, in conjunction with a detection scheme, which is optimal under the assumption of perfect synchronization. Since the reconstructed phase reference is only an approximation of the correct one, the overall detection scheme is only an approximation of ideal, i.e., with perfect phase reference, coherent detection. Although widely adopted, this solution should be regarded as just an ad-hoc heuristic procedure based on one possible logical approach and will be referred to as pseudocoherent.

Paper approved by E. Eleftheriou, the Editor for Equalization and Coding of the IEEE Communications Society. Manuscript received September 15, 1997; revised September 30, 1998 and February 3, 1999. This work was performed within a research cooperation between Dipartimento di Ingegneria dell'Informazionse, Università di Parma, Italy and Italtel S.p.A., Milano Italy. This paper was presented in part at the International Conference on Communications (ICC '97), Montreal, Canada, June 1997, and the International Conference on Universal Personal Communications (ICUPC '98), Florence, Italy, October 1998.

The authors are with the Dipartimento di Ingegneria dell'Informazione, Università di Parma, 43100 Parma, Italy.

Publisher Item Identifier S 0090-6778(99)07469-3.
Contrary to the above approach, noncoherent sequence detection provides us with optimal receivers, in the sense of minimum probability of erroneously detecting the entire information sequence, under a random time-invariant phase model. This paper adopts this viewpoint and proposes general receiver structures for approximating noncoherent sequence detectors, which are characterized by a performance close to that of an ideal coherent detector with affordable complexity. In principle, the time-invariant phase model is a key assumption because the information contained in the received signal may be shown to be unaffected by such a phase uncertainty, provided a sufficiently long observation is available. On the other hand, the approximations involved in the derivation of the proposed detection schemes have the convenient side-effect of allowing time-varying phase models.

The proposed detection schemes are applicable to linear or nonlinear coded modulations. In this paper, the case of coded linear modulations transmitted through AWGN channels possibly affected by intersymbol interference (ISI) is considered, whereas an extension to continuous phase modulations (CPM) is presented in a companion paper [1]. Being noncoherent, the proposed schemes do not have all the drawbacks of pseudocoherent detection, typically based on a phase-locked loop (PLL), such as acquisition problems, hangups, sensitivity to phase jitter, cycle slips, false locks, losses of lock caused by severe fading or oscillator frequency instabilities, etc. [2]-[5]. This paper expands upon previous work reported in [6]-[9].

Differential detectors, the simplest noncoherent receivers, are frequently employed to detect $M$-ary phase-shift keying ( $M$-PSK) modulations, with good performance only in the case of binary signaling (BPSK) [10]. Although differential detection eliminates the need for carrier acquisition and tracking, it suffers from a performance penalty when compared to ideal coherent detection. Some improvements are proposed in [11]-[14] for PSK modulations, using an extended differential detector based on decision feedback. These structures may be interpreted as special cases of the proposed detection schemes.

The performance of ideal coherent detection may be approached by more complex noncoherent receivers based on multiple-symbol differential detection. This approach was presented in [15]-[17] for PSK modulation (see also [18]) and extended to trellis-coded PSK [19]-[21], coded and uncoded quadrature amplitude modulation (QAM) and fading channels [22], and to improve the performance of PLL-based receivers [23]. Multiple-symbol differential receivers are based on maximum-likelihood detection of a block of information symbols based on a finite-duration signal observation.

A trellis-based noncoherent detection scheme is considered in [24], in which convolutional codes specifically tailored 


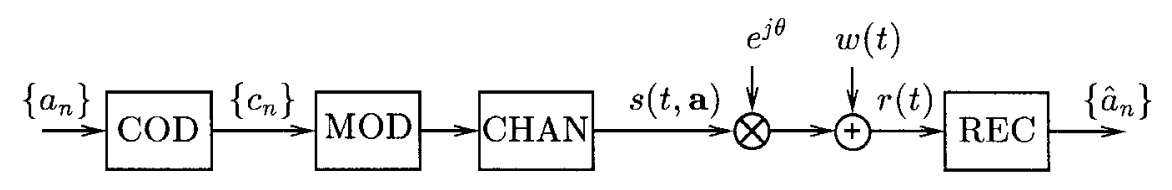

Fig. 1. System model.

for constant envelope modulations and noncoherent decoding are proposed. Noncoherent receivers therein utilized make use of a Viterbi algorithm (VA) whose branch metrics are heuristically assumed equal to the block metrics in multiplesymbol differential detection. This approximation is expected to limit the receiver performance, although this aspect is not directly addressed in [24]. Other trellis-based noncoherent schemes for QAM and PSK are presented in [25], [26], respectively, where the authors propose approximations of the optimal sequence metric. An approach similar to one here proposed for PSK may be found in seminal form in [26].

As a general result of most of the above bibliographical references, the performance of noncoherent detection schemes based on extended observation windows improves for increasing observation length and receiver complexity and approaches that of optimal coherent detection (as an example, see [16]).

The schemes proposed in this paper belong to the class of trellis-based noncoherent receivers, which overcome some limitations of block-by-block multiple-symbol differential receivers. With respect to other schemes, they are characterized by the fact that a performance gain may be achieved with acceptable levels of complexity-the tradeoff between complexity and performance being simply controlled by a parameter, referred to as implicit phase memory, and the number of trellis states. To our knowledge, the proposed schemes are the first to systematically and effectively solve the problem of noncoherent detection of nonequal energy signals such as coded QAM. The presented approach is very general and extends to the case of ISI-affected channels and CPM, for which a whitened matched filter (WMF) front-end is found to be practically essential. Complexity reduction techniques may also be employed by means of reduced-state sequence detection (RSSD) [27], [28].

In the next section, we review noncoherent sequence detection. The realization of this detection strategy entails a maximization of a suitable sequence metric by an exhaustive method. Alternatively, this maximization may be performed by a tree search algorithm, whose computational load grows exponentially with time. In Section III, we introduce some approximations in order to derive detection schemes suitable to be realized by means of a trellis search algorithm, such as a VA. In Section IV, these results are extended to account for the possible presence of ISI. Examples of applications for uncoded and coded linear modulations are considered in Section V. In Section VI, numerical results are presented. For increasing complexity, the performance of the proposed detection schemes is shown to approach that of optimal coherent receivers. A theoretical analysis of the performance of the proposed receivers for the special case of $M$-PSK, presented in [29], is also used. Finally, conclusions are drawn in Section VII.

\section{REVIEW OF NONCOHERENT SEQUENCE DETECTION}

The assumed system model is shown in Fig. 1. The information sequence $\left\{a_{n}\right\}$, composed of independent and identically distributed symbols belonging to an $M$-ary alphabet, is mapped into a code sequence $\left\{c_{n}\right\}$ by means of some coding rule. This code sequence is further mapped by a modulator in cascade with a channel filter into a time-continuous signal with complex envelope $s(t, \mathbf{a})$, which depends on the information sequence denoted by the vector a. This signal undergoes a phase rotation $\theta$ and is transmitted over an AWGN channel modeled by a complex-valued Gaussian white noise process $w(t)$ with independent components, each with twosided power spectral density $N_{0}$. The complex envelope of the received signal may be expressed as

$$
r(t)=s(t, \mathbf{a}) e^{j \theta}+w(t) .
$$

The phase rotation $\theta$ is modeled as a random variable with uniform distribution in the interval $[0,2 \pi)$. Hence, it is assumed to be constant during the entire transmission. The optimal noncoherent sequence detection strategy may be derived as described in [10, Appendix IV-C] and expressed as

$$
\begin{aligned}
\hat{\mathbf{a}}=\arg \max _{\tilde{\mathbf{a}}}\left\{-\frac{1}{2 N_{0}} \int_{T_{0}}|s(t, \tilde{\mathbf{a}})|^{2} d t\right. \\
\left.\quad+\log I_{0}\left(\frac{1}{N_{0}}\left|\int_{T_{0}} r(t) s^{*}(t, \tilde{\mathbf{a}}) d t\right|\right)\right\}
\end{aligned}
$$

in which $\hat{\mathbf{a}}$ is the detected sequence, $I_{0}(x)$ is the zeroth-order modified Bessel function of the first kind, $T_{0}$ is the observation interval, and $\tilde{\mathbf{a}}$ is a hypothetical information sequence. An alternative interpretation of the above noncoherent detection strategy is given in the Appendix.

\section{Noncoherent SEQuence Detection OF CODED Linear MOdUlations}

The strategy (2) is valid for any modulation format. In the case of linearly modulated signals, the information-bearing signal may be expressed as

$$
s(t, \mathbf{a})=\sum_{n=0}^{N_{T}-1} c_{n} h(t-n T)
$$

where $N_{T}$ denotes the number of transmitted code symbols, $T$ is the signaling interval, and $h(t)$ is a properly normalized shaping pulse. We now assume absence of ISI, i.e., $h(t) \otimes$ $\left.h^{*}(-t)\right|_{t=k T}=\delta_{k}$, where the symbol $\otimes$ denotes convolution and $\delta_{k}$ is the Kronecker delta. Substituting the signal model (3) in (2) and assuming a long enough observation interval $\left(T_{0} \rightarrow \infty\right)$, after some straightforward manipulations the 
detection strategy (2) becomes

$$
\begin{aligned}
\hat{\mathbf{a}}=\arg \max _{\tilde{\mathbf{a}}}\left\{-\frac{1}{2 N_{0}} \sum_{n=0}^{N_{T}-1}\left|\tilde{c}_{n}\right|^{2}\right. & \\
& \left.\quad+\log I_{0}\left(\frac{1}{N_{0}}\left|\sum_{n=0}^{N_{T}-1} x_{n} \tilde{c}_{n}^{*}\right|\right)\right\}
\end{aligned}
$$

where $\left\{\tilde{c}_{n}\right\}$ is the code sequence uniquely associated with the hypothetical information sequence ã by the given coding rule and, assuming perfect knowledge of symbol timing

$$
\left.x_{n} \triangleq r(t) \otimes h^{*}(-t)\right|_{t=n T}
$$

is the output, sampled at time $t=n T$, of a filter matched to the pulse $h(t)$. As in the case of known phase, the sampled output of a filter matched to the received pulse is a set of sufficient statistics. In the following, these samples will be referred to as "observations."

Although the metric used by detection strategy (4) is formally identical to (20) in [22], it is here obtained under the assumption of observing the time-continuous received signal $r(t)$ over an observation interval $T_{0}$ sufficiently longer than the message length $N_{T} T$, whereas in [18] it was obtained starting from a discrete signal model and with a limited observation window. As a consequence, the conceptual viewpoint is different.

The optimal noncoherent strategy (4) may be well approximated by letting $\log I_{0}(x) \simeq x$-the longer the transmission length, the better the approximation quality, for a given value of $N_{0}$. In the case of equal energy signals, such as PSK, this approximation is not necessary because the first term in (4), being constant for all sequences, can be omitted and $\log I_{0}(x)$ is a monotonically increasing function for $x \geq 0$.

We now consider the general case of nonequal energy signals. Let us define a partial sequence metric at the $n$th signaling interval

$$
\Lambda_{n}(\tilde{\mathbf{a}}) \triangleq-\frac{1}{2} \sum_{k=0}^{n-1}\left|\tilde{c}_{k}\right|^{2}+\left|\sum_{k=0}^{n-1} x_{k} \widetilde{c}_{k}^{*}\right|
$$

and an incremental metric

$$
\begin{aligned}
\Delta_{n}(\tilde{\mathbf{a}}) & \triangleq \Lambda_{n+1}(\tilde{\mathbf{a}})-\Lambda_{n}(\tilde{\mathbf{a}}) \\
& =\left|\sum_{k=0}^{n} x_{k} \tilde{c}_{k}^{*}\right|-\left|\sum_{k=0}^{n-1} x_{k} \tilde{c}_{k}^{*}\right|-\frac{1}{2}\left|\tilde{c}_{n}\right|^{2} .
\end{aligned}
$$

Obviously, the sequence metric $\Lambda_{N_{T}}(\tilde{\mathbf{a}})$ to be maximized may be recursively computed.

The difficulty inherent in the incremental metric (7) is its unlimited memory. In fact, this metric depends on the entire previous code sequence. This implies that the maximization of the sequence metric may, in principle, be realized by a search on a properly defined tree diagram. From the implementation viewpoint, approximate tree search algorithms must be used, unless a very short transmission length is assumed.

In order to limit the memory of the incremental metric (7), a truncation is introduced, aimed at allowing us to search a trellis diagram by means of a VA. To this end, in (7) we may consider $N \ll N_{T}$ most recent observations $x_{k}$ and code symbols $\tilde{c}_{k}$ only. After an initial transient period, i.e., for $n \geq N-1$, the resulting approximate truncated-memory incremental, or branch, metric is ${ }^{1}$

$$
\lambda_{n}(\tilde{\mathbf{a}}) \triangleq\left|\sum_{i=0}^{N-1} x_{n-i} \tilde{c}_{n-i}^{*}\right|-\left|\sum_{i=1}^{N-1} x_{n-i} \tilde{c}_{n-i}^{*}\right|-\frac{1}{2}\left|\tilde{c}_{n}\right|^{2} .
$$

We note that the approximation $\log I_{0}(x) \simeq x$ could have been avoided. By memory truncation, we would have obtained branch metrics

$$
\begin{aligned}
\lambda_{n}^{\prime}(\tilde{\mathbf{a}}) \triangleq & \log I_{0}\left(\frac{1}{N_{0}}\left|\sum_{i=0}^{N-1} x_{n-i} \tilde{c}_{n-i}^{*}\right|\right) \\
& -\log I_{0}\left(\frac{1}{N_{0}}\left|\sum_{i=1}^{N-1} x_{n-i} \tilde{c}_{n-i}^{*}\right|\right)-\frac{1}{2 N_{0}}\left|\tilde{c}_{n}\right|^{2}
\end{aligned}
$$

evidently dependent on the signal-to-noise ratio (SNR). These alternative metrics have been verified to be equivalent to (8), in terms of performance, in all the cases considered in Section VI.

In the case of equal energy signals, this approach leads to an incremental metric which differs from (8) in two respects: 1) the last term is absent; and 2) it is approximate because of memory truncation only. In this case, a different approach may also be derived. Since $x^{2}$ is a monotonically increasing function for $x \geq 0$, an equivalent noncoherent sequence metric is

$$
\begin{aligned}
\left|\sum_{n=0}^{N_{T}-1} x_{n} \tilde{c}_{n}^{*}\right|^{2} & =\sum_{n=0}^{N_{T}-1} \sum_{m=0}^{N_{T}-1} x_{n} x_{m}^{*} \tilde{c}_{n}^{*} \tilde{c}_{m} \\
& =\sum_{n=0}^{N_{T}-1}\left|x_{n}\right|^{2}\left|\tilde{c}_{n}\right|^{2}+2 \operatorname{Re}\left\{\sum_{n=1}^{N_{T}-1} x_{n} \tilde{c}_{n}^{*} \sum_{m=0}^{n-1} x_{m}^{*} \tilde{c}_{m}\right\}
\end{aligned}
$$

in which the metric has been expressed as a sum of all the elements of an $N_{T} \times N_{T}$ Hermitian matrix with elements $x_{n} x_{m}^{*} \tilde{c}_{n}^{*} \widetilde{c}_{m}$. The first sum in (10) is independent of the code sequence because $\left|\tilde{c}_{n}\right|^{2}=1$. Thus, an equivalent sequence metric is

$$
\Gamma_{N_{T}}(\tilde{\mathbf{a}}) \triangleq \operatorname{Re}\left\{\sum_{n=1}^{N_{T}-1} x_{n} \tilde{c}_{n}^{*} \sum_{m=0}^{n-1} x_{m}^{*} \tilde{c}_{m}\right\} .
$$

In order to limit the memory, a truncated incremental metric may be defined by a procedure similar to that used to derive (8) according to (for $n \geq N-1$ )

$$
\begin{aligned}
\gamma_{n}(\tilde{\mathbf{a}}) & \triangleq \operatorname{Re}\left\{x_{n} \tilde{c}_{n}^{*} \sum_{m=n-N+1}^{n-1} x_{m}^{*} \tilde{c}_{m}\right\} \\
& =\operatorname{Re}\left\{\sum_{i=1}^{N-1} x_{n} x_{n-i}^{*} \tilde{c}_{n}^{*} \tilde{c}_{n-i}\right\} .
\end{aligned}
$$

The effect of memory truncation is that the maximization of the sequence metric may be performed recursively on a properly defined trellis diagram using a VA with branch

\footnotetext{
${ }^{1}$ In the initial transient period, the expression of the branch metrics may remain unchanged if $x_{k}=0$ for $k<0$ is further assumed.
} 


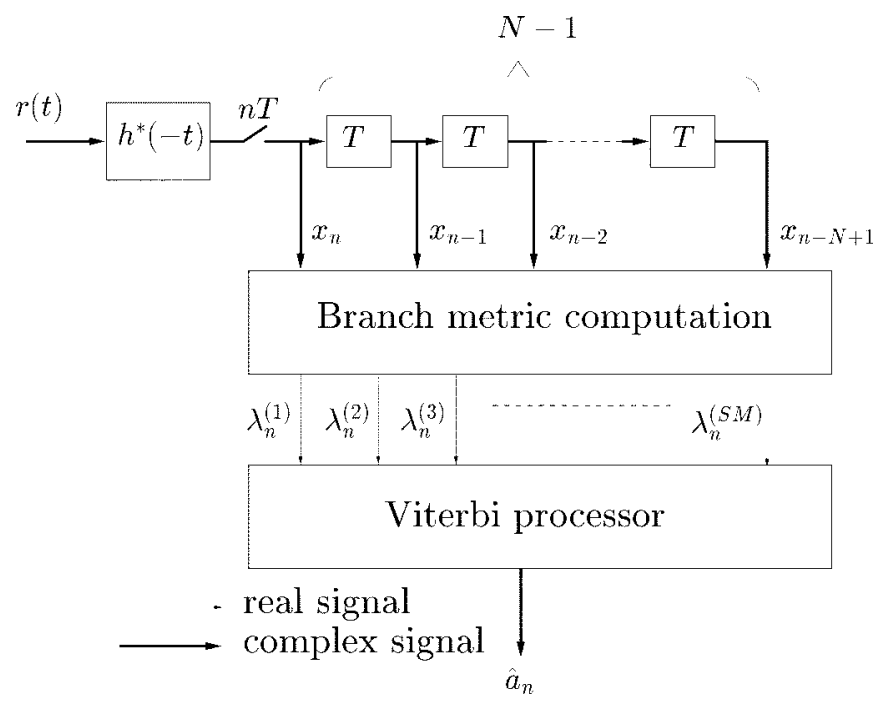

Fig. 2. Structure of the proposed noncoherent receiver.

metrics (8), or (12) for equal energy signals. For a given coding rule, the code symbols $\left\{c_{n}\right\}$ may be expressed in terms of the information symbols $\left\{a_{n}\right\}$ and a trellis state defined accordingly. Since (8) and (12) depend on $N$ code symbols, in general, the number of trellis states is larger than that of the code trellis. However, this complexity increase may be limited by a possible use of techniques for state-complexity reduction, in order to limit the number of states without excessively reducing the value of $N$, as described at the end of this section. Even using small values of $N$ (a few units), a performance very close to that of coherent detection may be obtained, as shown in the numerical results. In the limit as $N \rightarrow+\infty$, coherent detection performance is obtained. A block diagram of the baseband equivalent model of the proposed receivers is shown in Fig. 2.

We now comment on combined demodulation and decoding of coded linear modulations. From (4), it may be noted that two code sequences which differ for a constant phase shift have the same noncoherent metric and are indistinguishable. For this reason, a code such that different code sequences differ only for a constant phase shift is noncoherently catastrophic (NC) [24]. The usual rotationally invariant (RI) codes are NC codes. Noncoherent decoding of these codes is possible only if they are used in conjunction with a differential encoder, which makes the total code noncoherently noncatastrophic. On the other hand, a noncoherent receiver can decode a code that does not satisfy rotationally invariance (NRI code) provided it is not NC. Some examples of trellis-coded modulations (TCM's) presented in [30], [31] are not NC codes and are explicitly considered in the numerical results. For this purpose, the trellis diagram has to be defined in terms of the information symbols and, in the case of a differentially encoded RI scheme, the VA also implements differential decoding. This is a fundamental structural difference with respect to PLL-based pseudocoherent systems using RI codes with differential encoding, ${ }^{2}$ in which the trellis diagram must be defined in terms of code symbols or,

\footnotetext{
${ }^{2}$ The use of an NRI code in a pseudocoherent receiver may cause a long acquisition period in decision-directed phase synchronizers, as well as phase ambiguity problems in nondata-aided phase synchronizers.
}

equivalently, differentially encoded symbols, and an external differential decoder is required to recover the information sequence.

In order to provide some interpretations, we concentrate on $M$-PSK and consider the second algorithm with branch metrics (12). A coherent receiver for coded $M$-PSK selects the sequence $\left\{c_{n}\right\}$ that maximizes the sum of branch metrics $\operatorname{Re}\left\{x_{n} \tilde{C}_{n}^{*} e^{-j \theta}\right\}$ in which $\theta$ is the correct channel phase [10]. It is easy to verify that an ML data-aided estimate of the phase $\theta$ based on the observation of signal $x_{k}$ in (5), where $k=0,1, \cdots, n-1$, satisfies

$$
e^{-j \hat{\theta}}=\frac{\sum_{m=0}^{n-1} x_{m}^{*} c_{m}}{\left|\sum_{m=0}^{n-1} x_{m}^{*} c_{m}\right|} \simeq \frac{1}{n} \sum_{m=0}^{n-1} x_{m}^{*} c_{m}
$$

where the last approximation holds for low noise because the denominator is approximately a constant. ${ }^{3}$ The approximation (13) can be recognized as proportional to the inner sum in (11) for $\tilde{c}_{n}=c_{n}$. Hence, this inner sum may be interpreted as a phase estimate based on per-survivor processing (PSP) [33]. As a consequence, memory truncation affects the quality of the phase estimate which is implicit in the expression of the branch metrics (12). As shown in the following sections, the resulting performance loss is limited. We thus have an intuitive understanding of the fact that for $N \rightarrow+\infty$, the performance tends to that of coherent detection. Integer $N$ affects the number of previous symbols that aid an implicit per-survivor phase estimator and is intimately related to the definition of trellis state. For this reason, we refer to $N$ as implicit phase memory parameter.

Based on this interpretation, alternative noncoherent detection schemes may be devised for nonequal energy linearly modulated signals [8] and CPM (see [1] and references therein).

Let us now consider a time-varying phase model. The proposed algorithms require an approximately constant channel phase in a window of $N$ symbol intervals only. In this case, an estimation only based on the most recent observations has greater accuracy. In fact, under dynamic channel conditions, the receiver sensitivity to the time-varying phase increases with the phase memory $N$ because the phase of term $x_{k} x_{k-N+1}^{*}$, which appears in the expression of the branch metrics (12), may vary due to the channel phase variations. Similar considerations can be made for the algorithm based on (8).

There is an analogy between the proposed receiver based on branch metrics (12) and the multiple-symbol differential receiver for $M$-PSK [16]. In [16], the receiver is based on an exhaustive search on a block of $N$ symbols. Proceeding as in the derivation of (11), the detection strategy of [16] may be expressed as

$$
\hat{\mathbf{c}}_{N}(k)=\underset{\tilde{\mathbf{c}}_{N}(k)}{\operatorname{argmax}}\left\{\operatorname{Re}\left[\sum_{n=k N-N+2}^{k N} x_{n} \tilde{c}_{n}^{*} \sum_{m=k N-N+1}^{n-1} x_{m}^{*} \tilde{c}_{m}\right]\right\}
$$

\footnotetext{
${ }^{3}$ The right-hand-side in (13) is the exact ML estimate of the complex number $e^{-j \theta}$. This viewpoint suggests a relation with vector tracking [32].
} 
where $\mathbf{c}_{N}(k)$ is the $k$ th block. The inner sum may be interpreted as a phase estimate based on a number of samples $x_{m}$, which increases from 1 to $N-1$ as long as the index $n$ scans the block from beginning to end. In our algorithm, we extend this number of terms to its maximum value $N-1$ for each $n$ (i.e., we are not constrained inside the block) and the outer sum to the whole transmission. We intuitively expect that our algorithm performs better when its phase memory equals the block length of the receiver in [16], since its implicit phase estimate is more accurate. This is confirmed by the numerical results shown in Section VI.

The state-complexity of the proposed detection schemes may be limited by RSSD [27], [28]. As an example, considering a trellis state defined in terms of $\zeta$ information symbols as $\sigma_{n} \triangleq\left(\tilde{a}_{n-1}, \tilde{a}_{n-2}, \cdots, \tilde{a}_{n-\zeta}\right)$, the number of states is $S=M^{\zeta}$. A reduced state $\sigma_{n}^{\prime} \triangleq\left(\tilde{a}_{n-1}, \tilde{a}_{n-2}, \cdots, \tilde{a}_{n-\beta}\right)$ with $\beta<\zeta$ may be defined. The resulting number of states is reduced to $M^{\beta}<M^{\zeta}$. More complex techniques based on set partitioning may also be employed [27], [28]. In order to compute the branch metrics (8) or (12) in a reduced trellis, the necessary symbols not included or not completely specified in the state definition may be found in the survivor history according to PSP [33]. We note that, in the limiting case of $\beta=0$, the trellis diagram degenerates and symbol-by-symbol detection with decision feedback is performed. Therefore, the receivers proposed in [11]-[14] for PSK are a special case of the proposed detection schemes.

\section{Noncoherent Sequence Detection of Coded Linear MOdUlations IN THE PRESENCE OF ISI}

We now consider the case of a channel affected by ISI. Among various possible applications, this case has practical relevance for the realization of adaptive receivers for ISI channels and in systems using partial response signaling. This case is also the basis for the extension of noncoherent sequence detection to CPM [1].

Defining $g_{k} \triangleq g(k T)$, where $g(t) \triangleq h(t) \otimes h^{*}(-t)$ is the overall channel impulse response, and assuming perfect symbol timing, the detection strategy (2) becomes ${ }^{4}$

$$
\begin{aligned}
\hat{\mathbf{a}}=\underset{\tilde{\mathbf{a}}}{\operatorname{argmax}}\left\{-\frac{1}{2 N_{0}} \sum_{k=0}^{N_{T}-1} \sum_{n=0}^{N_{T}-1} \tilde{c}_{k} \tilde{c}_{n}^{*} g_{n-k}\right. \\
\left.\quad+\log I_{0}\left(\frac{1}{N_{0}}\left|\sum_{k=0}^{N_{T}-1} x_{k} \tilde{c}_{k}^{*}\right|\right)\right\}
\end{aligned}
$$

where $x_{k}$ is defined by (5). As shown by (15), the sampled output $\left\{x_{k}\right\}$ of a matched filter is a sufficient statistic for optimal noncoherent detection of the information sequence, as in the case of known phase [34].

Proceeding as in [35], an alternative set $\left\{z_{k}\right\}$ of sufficient statistics may be obtained through a WMF whose output may be expressed as

$$
z_{k}=y_{k} e^{j \theta}+w_{k}
$$

\footnotetext{
${ }^{4}$ In Section III, $g(t)$ satisfies the Nyquist criterion; hence, $g_{k}=\delta_{k}$.
}

in which $\left\{w_{k}\right\}$ are Gaussian zero-mean independent random variables of variance $\sigma_{w}^{2}=2 N_{0}$ and

$$
y_{k} \triangleq \sum_{l=0}^{L} f_{l} c_{k-l}
$$

where $L$ is a channel memory parameter and the equivalent time-discrete channel $\left\{f_{k}\right\}$ may be obtained from sequence $\left\{g_{k}\right\}$ following the method described in [35]. An alternative formulation of the optimal noncoherent strategy may be based on sequence $\left\{z_{k}\right\}$, denoted in the following by a vector z. Starting from the conditional probability density function $p(\mathbf{z} \mid \tilde{\mathbf{a}}, \tilde{\theta})$ and averaging with respect to $\tilde{\theta}$, we obtain the relevant likelihood function $p(\mathbf{z} \mid \tilde{\mathbf{a}})$ for optimal noncoherent detection. The resulting detection strategy may be expressed as

$$
\hat{\mathbf{a}}=\operatorname{argmax}\left\{-\frac{1}{2 N_{0}} \sum_{k=0}^{N_{T}-1}\left|\tilde{y}_{k}\right|^{2}+\log I_{0}\left(\frac{1}{N_{0}}\left|\sum_{k=0}^{N_{T}-1} z_{k} \tilde{y}_{k}^{*}\right|\right)\right\}
$$

in which $\tilde{y}_{k}$ is defined in terms of the hypothetical code sequence $\left\{\tilde{c}_{k}\right\}$ as in (17).

As in the case of absence of ISI, the equivalent optimal noncoherent sequence metrics (15) and (18) may be exactly maximized by an exhaustive method whose complexity increases exponentially with $N_{T}$. With the same approximations used in Section III, the truncated-memory incremental metrics relative to strategy (15) are

$$
\begin{aligned}
\lambda_{n}^{U}(\tilde{\mathbf{a}}) \triangleq \mid & \left|\sum_{i=0}^{N-1} x_{n-i} \tilde{c}_{n-i}^{*}\right|-\left|\sum_{i=1}^{N-1} x_{n-i} \tilde{c}_{n-i}^{*}\right| \\
& -\frac{1}{2}\left\{\left|\tilde{c}_{n}\right|^{2} g_{0}+2 \operatorname{Re}\left[\sum_{l=1}^{L} \tilde{c}_{n} \tilde{c}_{n-l}^{*} g_{l}^{*}\right]\right\}
\end{aligned}
$$

whereas those relative to strategy (18) are

$$
\lambda_{n}^{F}(\tilde{\mathbf{a}}) \triangleq\left|\sum_{i=0}^{N-1} z_{n-i} \tilde{y}_{n-i}^{*}\right|-\left|\sum_{i=1}^{N-1} z_{n-i} \tilde{y}_{n-i}^{*}\right|-\frac{1}{2}\left|\tilde{y}_{n}\right|^{2} .
$$

The effect of the introduced memory truncation is that the maximization of the sequence metric may be performed recursively by searching a properly defined trellis diagram using a VA with branch metrics (19) or (20), respectively.

Even if the noncoherent receivers with branch metrics (19) and (20) are derived from two equivalent formulations of the optimal noncoherent strategy, they do not have comparable performance because the introduced approximations have very different effects in the two cases. In strategy (15) (referred to as Ungerboeck approach), each sample $x_{k}$ is correlated with a corresponding code symbol $c_{k}$, whereas in strategy (18) (referred to as Forney approach), each sample $z_{k}$ is correlated with $y_{k}$. In the latter strategy, sequences $\left\{z_{k}\right\}$ and $\left\{y_{k}\right\}$ differ for a sequence of independent noise samples $\left\{w_{k}\right\}$ only [see (16)]. On the other hand, in the former strategy, the corresponding sequences $\left\{x_{k}\right\}$ and $\left\{c_{k}\right\}$ are significantly more different because of ISI and noise correlation. These effects tend to cancel out in the sequence metric (15) (summation extended over the entire transmission), but are significant in 
the branch metrics (19) (truncated summation). For a given and moderate complexity, the suboptimal receiver based on the Forney approach performs significantly better. In the numerical results, we concentrate on this approach.

\section{EXAMPLES OF APPLICATIONS}

We describe some examples of applications in the case of absence of ISI.

\section{A. Differentially Encoded QAM}

In this section, we assume symbols $\left\{c_{n}\right\}$ belong to an $M$ ary square QAM alphabet and are derived from information symbols $\left\{a_{n}\right\}$, belonging to the same alphabet, by means of the following quadrant differential encoding rule [36]. The generic information symbol is uniquely represented as $a_{n}=\mu_{n} p_{n}$, where $\mu_{n}$ belongs to the first quadrant and $p_{n} \in\{ \pm 1, \pm j\}$. The encoded symbol $c_{n}$ is given by $c_{n}=\mu_{n} q_{n}$, where $q_{n} \in\{ \pm 1, \pm j\}$ is defined by $q_{n}=p_{n} q_{n-1}$, i.e., the usual differential encoding rule for quadrature phase shift keying (QPSK) modulations applied to symbols $\left\{p_{n}\right\}$.

The branch metrics (8) must be expressed in terms of the information symbols in such a way that the VA also implements differential decoding. Multiplying the terms $\left|\sum_{i=0}^{N-1} x_{n-i} \tilde{c}_{n-i}^{*}\right|$ and $\left|\sum_{i=1}^{N-1} x_{n-i} \tilde{c}_{n-i}^{*}\right|$ by $\left|\tilde{q}_{n}\right|=1$, noting that ${ }^{5}$

$$
\tilde{q}_{n} \tilde{c}_{n-i}^{*}=\tilde{\mu}_{n-i}^{*} \tilde{q}_{n-i}^{*} \tilde{q}_{n}=\tilde{\mu}_{n-i}^{*} \prod_{m=0}^{i-1} \tilde{p}_{n-m}
$$

and $\left|\tilde{c}_{n}\right|=\left|\tilde{a}_{n}\right|=\left|\tilde{\mu}_{n}\right|$, we obtain

$$
\begin{aligned}
\lambda_{n}(\tilde{\mathbf{a}})=\left|\sum_{i=0}^{N-1} x_{n-i} \tilde{\mu}_{n-i}^{*} \prod_{m=0}^{i-1} \tilde{p}_{n-m}\right| \\
-\left|\sum_{i=1}^{N-1} x_{n-i} \tilde{\mu}_{n-i}^{*} \prod_{m=0}^{i-1} \tilde{p}_{n-m}\right|-\frac{1}{2}\left|\tilde{a}_{n}\right|^{2} .
\end{aligned}
$$

According to (22), a trellis state may be defined as

$$
\begin{aligned}
\sigma_{n} & \triangleq\left(\tilde{\mu}_{n-1}, \tilde{\mu}_{n-2}, \cdots, \tilde{\mu}_{n-N+1}, \tilde{p}_{n-1}, \tilde{p}_{n-2} \cdots, \tilde{p}_{n-N+2}\right) \\
& =\left(\tilde{a}_{n-1}, \tilde{a}_{n-2}, \cdots, \tilde{a}_{n-N+2}, \tilde{\mu}_{n-N+1}\right) .
\end{aligned}
$$

The number of states $S=\frac{1}{4} M^{N-1}$ depends exponentially on integer $N$. Complexity reduction techniques may also be adopted.

A noncoherent receiver for QAM based on multiple-symbol differential detection was proposed in [22]. A different type of differential encoding was adopted there, which, however, modifies an original $M$-point square QAM constellation into one characterized by a larger number of points. Our tests indicate that quadrant differential encoding does not ensure satisfactory performance when applied to the receiver in [22] - the reason being related to the strictly limited observation window there assumed.

\footnotetext{
${ }^{5}$ Symbols $\tilde{\mu}_{n}, \tilde{p}_{n}$, and $\tilde{q}_{n}$ are defined by this differential encoding rule applied to the hypothetical data sequence $\left\{\tilde{a}_{n}\right\}$.
}

\section{B. Differentially Encoded PSK}

In this section, we assume that symbols $\left\{c_{n}\right\}$ belong to an $M$-PSK alphabet and are derived from symbols $\left\{a_{n}\right\}$, belonging to the same alphabet, by means of the differential encoding rule $c_{n}=c_{n-1} a_{n}$. In this case, the branch metrics (8) and (12) become, respectively

$$
\begin{aligned}
& \lambda_{n}(\tilde{\mathbf{a}})=\left|\sum_{i=0}^{N-1} x_{n-i} \prod_{m=0}^{i-1} \tilde{a}_{n-m}\right|-\left|\sum_{i=1}^{N-1} x_{n-i} \prod_{m=0}^{i-1} \tilde{a}_{n-m}\right| \\
& \gamma_{n}(\tilde{\mathbf{a}})=\operatorname{Re}\left\{\sum_{i=1}^{N-1} x_{n} x_{n-i}^{*} \prod_{m=0}^{i-1} \tilde{a}_{n-m}^{*}\right\}
\end{aligned}
$$

having expressed the code symbols $\left\{c_{n}\right\}$ in terms of the information symbols $\left\{a_{n}\right\}$, using the differential encoding rule. According to (24) or (25), a trellis state may be properly defined as $\sigma_{n} \triangleq\left(\tilde{a}_{n-1}, \tilde{a}_{n-2}, \cdots, \tilde{a}_{n-N+2}\right)$. The number of states $S=M^{N-2}$ depends exponentially on $N$, although techniques for complexity reduction may be used.

\section{Noncoherent Coded Modulations}

In [24], the problem of noncoherent sequence detection and decoding is approximately solved by a search on a trellis diagram whose branch metrics are heuristically defined similarly to the block metrics in [16]. Under this suboptimal assumption on the detection scheme, optimal convolutional codes for $M$-PSK signaling were derived in [24] by an exhaustive search. The resulting scheme is called noncoherent coded modulation.

Here, we approach the decoding of noncoherent coded modulations by the schemes previously introduced. We assume the encoder structure described in [24]. Let $\nu$ be the code constraint length and $\eta$ the number of code symbols per information symbol. As in [24], we consider encoder structures that consist of only one shift register with code rate $1 / \eta$. Each information symbol $a_{k}$ generates $\eta$ code symbols $\left\{c_{\eta k+l}\right\}$ with $l=0,1, \cdots, \eta-1$. Information and code symbols belong to the $M$-PSK alphabet. The number of code states is $S_{c}=M^{\nu-1}$. Using the branch metrics (8) or (12), the number of trellis states of the proposed receivers is $S=S_{C} M^{N / \eta-1}$.

\section{Trellis-Coded Modulations}

The proposed noncoherent schemes may be used in the decoding of TCM. As in the previous cases, the branch metrics must be expressed in terms of the information symbol sequence, given the coding rule. A general explicit expression would require an involved notation and is not pursued. Specific codes are considered in the numerical results.

\section{E. Pilot-Symbol-Assisted Systems}

As an alternative to differential encoding, the problem of impossible recovery of an absolute phase reference may be avoided using a pilot symbol periodically inserted in the transmitted data stream. The extension of the proposed receivers to detection using pilot symbols is straightforward: when the periodic known symbol is received, only a fraction $1 / M$ of the survivors is retained, i.e., only those of states that 


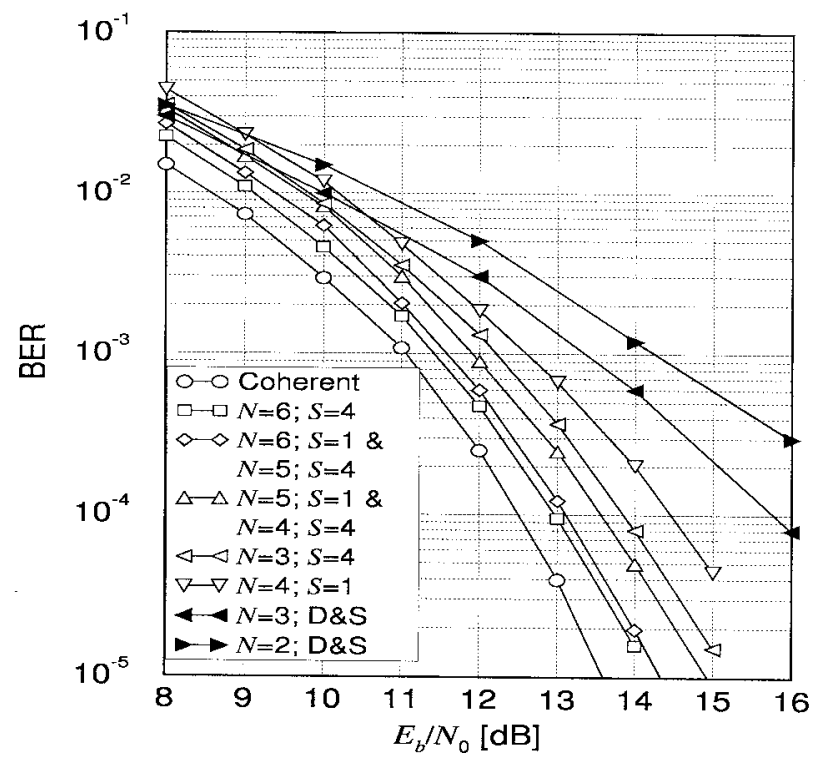

Fig. 3. BER of the proposed detection schemes for 16-DQAM with various degrees of complexity and comparison with the receiver in [22] (D\&S).

are compatible with the known pilot symbol. The remaining survivors are discarded by forcing their metric to very low values.

\section{NUMERICAL RESULTS}

The performance of the proposed receivers is assessed by means of computer simulation in terms of bit-error rate (BER) versus $E_{b} / N_{0}, E_{b}$ being the received signal energy per information bit. Besides "full-state" receivers, reducedstate techniques have also been considered. Unless otherwise stated, the channel phase is assumed constant. In the case of $M$-PSK, the theoretical analysis proposed in [29] is also used.

For differentially encoded 16-QAM (16-DQAM), we only consider schemes with a number of states $S=4$ (i.e., the state is defined as $\sigma_{n}^{\prime}=\tilde{\mu}_{n}$ ) and $S=1$ (i.e., symbolby-symbol detection with decision feedback is performed). Fig. 3 shows the relevant performance, obtained by computer simulation, for various values of $N$ and compares it with that of optimal coherent detection. The receivers proposed in [22] by Divsalar and Simon (D\&S in the figure) are also considered for comparison, assuming $N$ is the block length. It may be observed that the proposed receivers perform better and exhibit a loss of only $0.5 \mathrm{~dB}$ at a BER of $10^{-5}$ with respect to coherent detection, with affordable complexity. For larger complexity, the performance approaches that of coherent detection. We also note that the performance tends to that of coherent detection with a rate that is independent of the SNR.

Noncoherent sequence detection has also been applied to TCM. Two 8-state trellis-coded (TC) 16-QAM schemes, optimal under coherent detection, are considered: the first is an NRI code [30] and the second a $90^{\circ}$ RI code [31]. As already mentioned in Section III, the RI code is used in conjunction with a differential encoder and, in the proposed algorithms, the VA also implements differential decoding. Various noncoherent receivers with different complexity have been analyzed. Fig. 4 shows the performance of the considered

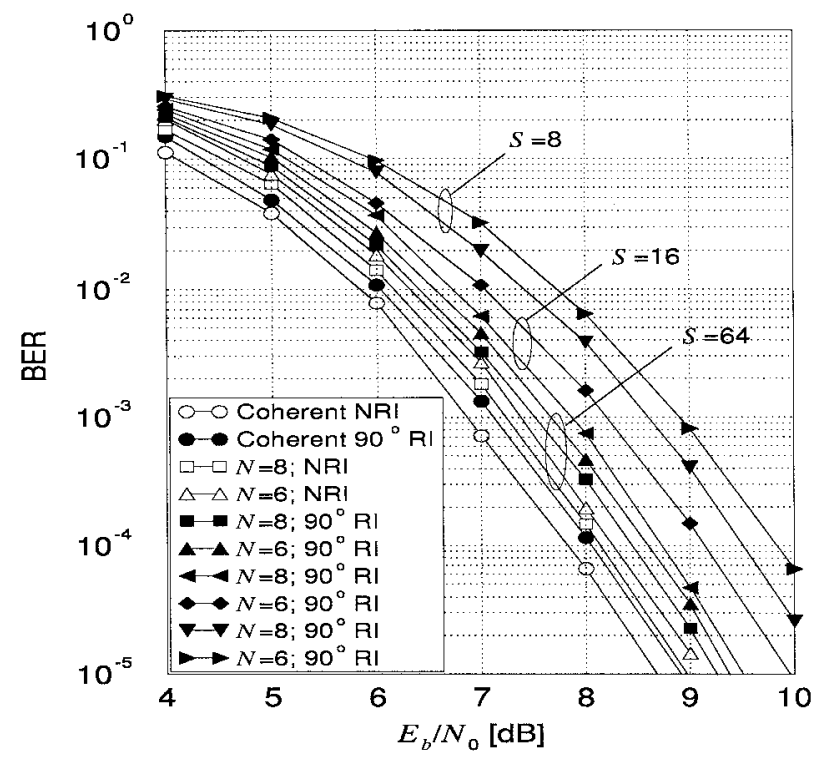

Fig. 4. BER of the proposed detection schemes for 8-state TC-16-QAM.

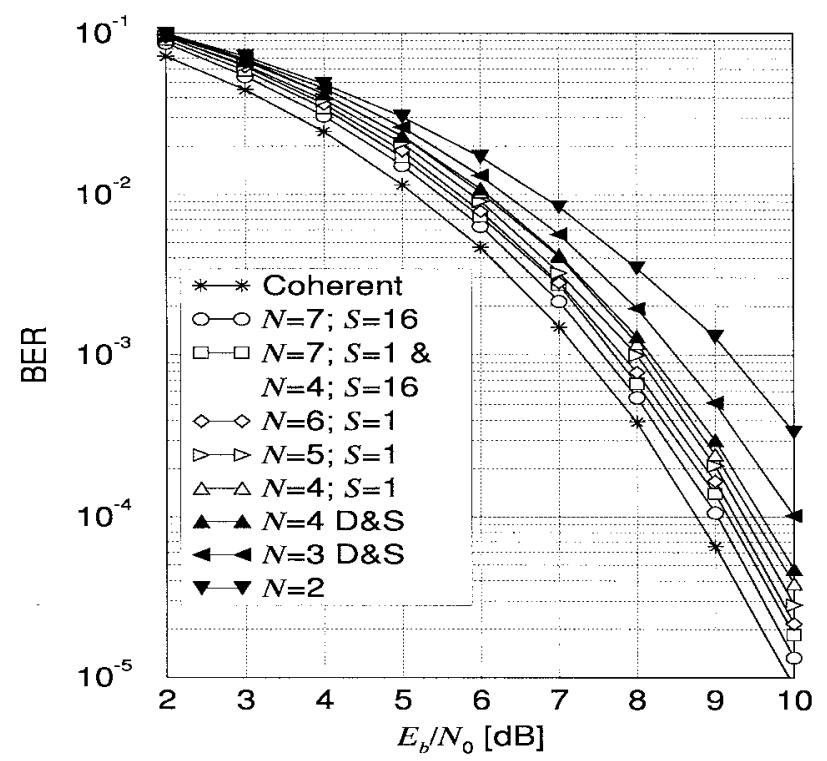

Fig. 5. BER of the proposed detection schemes for DQPSK (white marks) and comparison with receivers in [16] by D\&S (black marks).

receivers along with that of coherent detection. Receivers based on the code trellis $(S=8)$ exhibit a performance loss of about $1.5 \mathrm{~dB}$ (for $N=8$ ), but with an increase in the number of states up to $S=64$ the performance loss becomes negligible. The state of the receivers with $S=16$ is defined by a complete representation of the previous information symbol $\tilde{a}_{n-1}$ and a partial representation of symbol $\tilde{a}_{n-2}$. We have also considered two 8-state TC-8-PSK schemes. The numerical results for these cases are similar.

Differentially encoded QPSK (DQPSK) is considered in Fig. 5. The performance of the proposed noncoherent receiver based on algorithm (12) is compared with that of the receiver proposed in [16] (D\&S in the figure). In this case, the performance of receivers based on algorithm (8) is not reported because it is very similar. As previously noted on the basis 


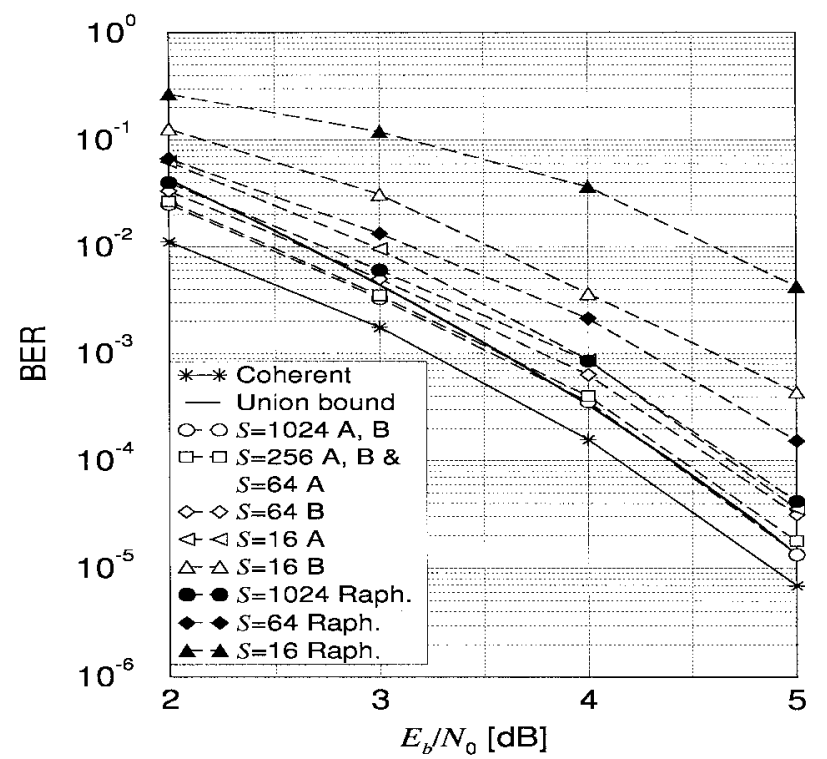

Fig. 6. Comparison, for noncoherent coded QPSK, between the proposed receiver (white marks) and the receivers in [24] by Raphaeli for $N=8$ (black marks). Algorithms (8) and (12) are denoted as A and B, respectively.

of intuitive remarks, the proposed receiver performs better when its phase memory parameter equals the block length of the receiver in [16] (both parameters are denoted by $N$ in the figure). For $N=4$, the proposed receiver with $S=16$ gains $0.5 \mathrm{~dB}$ with respect to the receiver in [16]. This gain becomes negligible if we consider the symbol-bysymbol decision-feedback receiver with $N=4$. For $N=2$, both receivers become the classical differential receiver and their performance coincides. Receivers with a state number larger than $S=16$ are not considered. To further increase the phase memory $N$, we apply the previously described statereduction techniques. We may note that the BER tends to that of coherent detection with differential encoding for increasing values of $N$. With a symbol-by-symbol receiver and $N=7$ the performance degradation with respect to ideal coherent detection is negligible $\left(0.4 \mathrm{~dB}\right.$ at a BER of $\left.10^{-5}\right)$.

The proposed noncoherent receivers, based on algorithms (8) and (12), have been compared with that proposed in [24]. In [24], the optimal code generators for the metrics therein presented are reported. Here, we have simply adopted from [24] the optimal code for QPSK with $\nu=3$ and $\eta=2\left(S_{c}=16\right)$, which, incidentally, has been verified to coincide with the optimal code for our schemes [29]. The code generators are $^{6} \mathbf{g}_{1}=(1,3,3)$ and $\mathbf{g}_{2}=(2,3,1)$ [24], and the code is NRI. A phase memory parameter equal to $N=8$ code symbols is considered. For all full-state receivers, the number of states is $S=1024$. The performance of the receiver in [24] for $S=1024$ may be found in [37]. We have simulated not only the full-state receivers, but also reduced-state ones. Fig. 6 shows the relevant results. Algorithms (8) and (12) (denoted in the figure as A and B, respectively) have equal performance when full-complexity receivers are considered. However, algorithm (8) has better performance when complexity reduction techniques are used.

\footnotetext{
${ }^{6}$ Base- 4 representation.
}

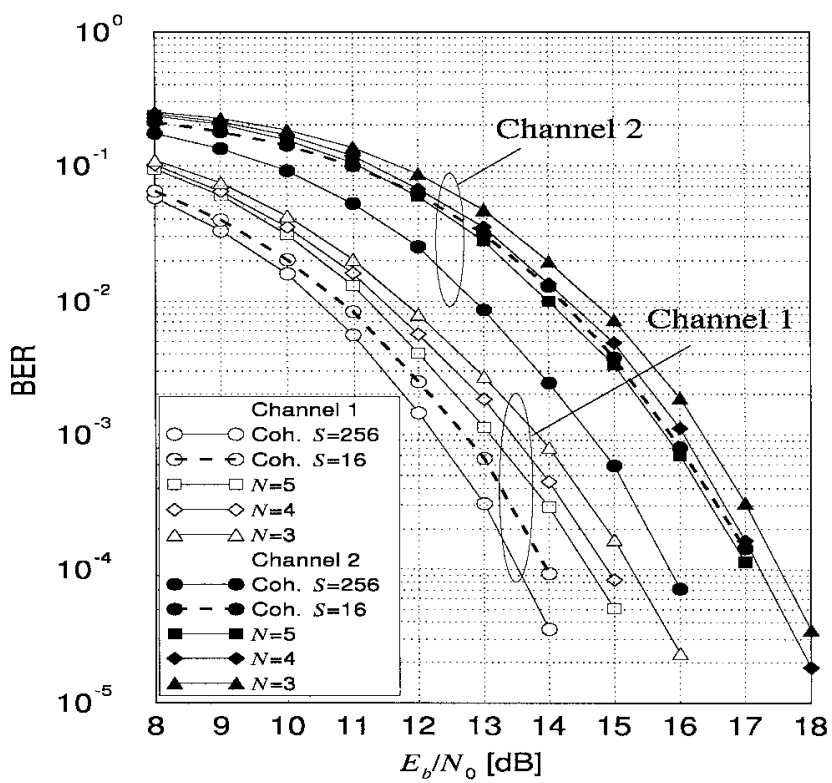

Fig. 7. BER of the proposed detection schemes for 16-DQAM on the two considered ISI channels and various values of $N$. The noncoherent detectors search a trellis with $S=256$ states.

As a comparison, we also show the performance of a coherent receiver with the optimal $90^{\circ} \mathrm{RI}$ code for coherent detection (with the same rate and constraint length) whose generators are $\mathbf{g}_{1}=(1,1,1)$ and $\mathbf{g}_{2}=(2,3,2)$. We may observe that the performance loss of the proposed schemes is about $0.2 \mathrm{~dB}$ at a BER of $10^{-5}$. As also shown in the figure, simulation results have been confirmed by the truncated union bound for the fullcomplexity receiver based on branch metrics (12) presented in [29]. As we may see from Fig. 6, the proposed receivers have better performance with respect to that in [24] for limited statecomplexity. A similar behavior has been observed for other convolutional codes and modulation formats.

We now consider the case of linear modulations in the presence of ISI. The receiver is composed of a WMF and a VA based on branch metrics (20). 16-DQAM and two channels with $L=2$ are considered. They correspond to the following overall discrete impulse responses at the output of the WMF: $\left(f_{0}, f_{1}, f_{2}\right)=(0.3,0.9,0.3)$ for channel 1 and $\left(f_{0}, f_{1}, f_{2}\right)=$ $(0.408,0.816,0.408)$ for channel 2 [10]. In this case, the optimal coherent receiver is characterized by $S=256$ states. In Fig. 7, the performance of the optimal coherent receiver for both channels is shown and compared with the performance of the proposed noncoherent sequence detection schemes with various values of $N$. For a given value of $N$, the number of states resulting from (20) is $S=\frac{1}{4} M^{N+L-1}=4 \cdot 16^{N}$. In the simulations, we used the described state-complexity reduction techniques and considered a number of states $S=256$, i.e., the same number of states of the optimal coherent receivers for the two channels. The state of these noncoherent receivers is defined by a complete representation of the symbols $\tilde{a}_{n-1}$ and $\tilde{a}_{n-2}$. Despite the constraint on state-complexity, for increasing values of $N$, the BER tends to approach that of coherent detection. State-complexity reduction has also been used in the coherent receiver. In the figure, the performance for $S=16$ states is shown for the considered channels. The state 


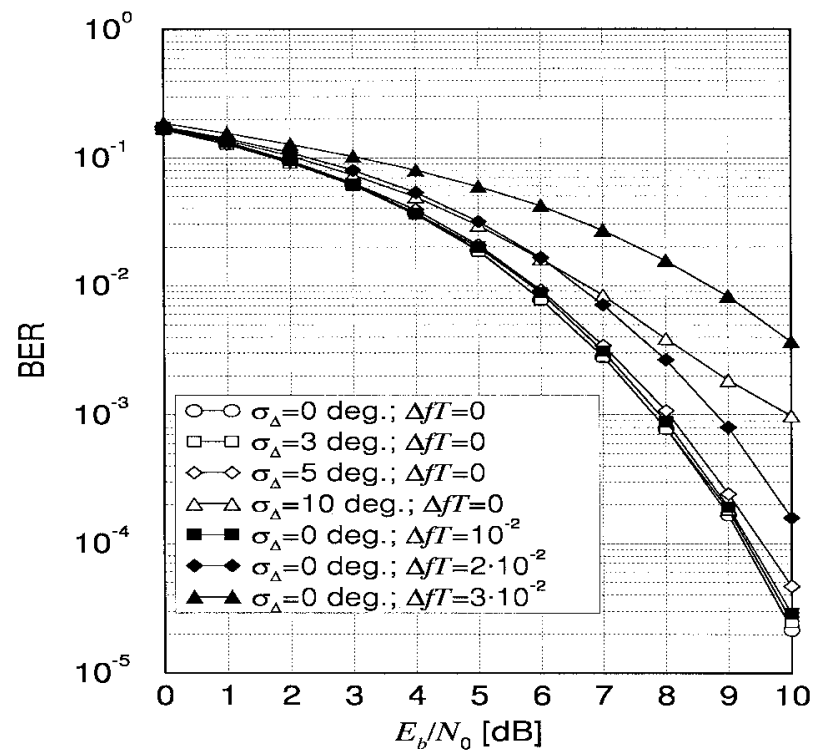

Fig. 8. BER of the proposed receiver for DQPSK with $N=6$ and $S=1$ for various values of phase jitter standard deviation (white marks) and frequency offset (black marks).

definition takes into account a complete representation of symbols $\widetilde{a}_{n-1}$ only. We then have a twofold comparison between coherent and noncoherent sequence detection. With reference to the proposed noncoherent receivers, the figure shows their performance loss, under an equal state-complexity constraint, and their complexity increase, under an approximate equalperformance constraint.

The performance under dynamic channel conditions has also been investigated, assuming the transmitter and receiver filters have square-root raised-cosine frequency response with rolloff 0.5. Two types of time-varying phase models are considered. The first is the well-known stochastic model of phase jitter. Accordingly, the phase $\theta$ of the received signal is modeled as a time-continuous Wiener process with incremental variance over a signaling interval equal to $\sigma_{\Delta}^{2}$. The second is a deterministic model of a frequency offset $\Delta f$. The proposed noncoherent receivers are robust to phase jitter and frequency offset, as may be observed in Fig. 8 for DQPSK, $N=6$, and $S=1$ [algorithm (12)]. A jitter standard deviation up to five degrees (per signaling interval) does not significantly degrade the receiver performance. Values of the frequency offset $\Delta f$, normalized to the symbol frequency $1 / T$, up to $\Delta f T=10^{-2}$ do not entail appreciable degradation.

\section{CONCLUSION}

In this paper, a class of algorithms for combined noncoherent detection and decoding of coded linear modulations have been presented. Various approximations have been considered, with the aim of limiting the inherently unlimited memory of properly defined incremental sequence metrics. Under these approximations, trellis diagrams that represent both the code memory and an implicit phase memory may be defined and searched by a VA. State-reduction techniques may also be employed. The tradeoff between performance and complexity may be controlled by an implicit phase memory parameter and the level of state-complexity reduction.

The proposed detection schemes have been assessed by computer simulation for several uncoded or coded linear modulations of practical significance. The case of ISI channels has also been addressed. These schemes have a performance that can be made arbitrarily close to that of coherent detection and compare favorably with other solutions previously proposed in the literature. Very good performance may be achieved with affordable complexity. Being noncoherent, they do not have all the drawbacks of conventional approximation of coherent detection based on the use of PPL and are especially attractive for burst-mode transmissions.

\section{APPENDIX}

Let us consider a deterministic unknown phase model. In this case, an optimal receiver cannot be defined because a uniformly most powerful test does not exist [38]. A logical procedure may be the "generalized likelihood" method [38], i.e., the simultaneous maximization of the joint likelihood function with respect to data and phase, for the given observation. This approach is followed in [11], where it is observed that for linear modulations, it is possible to detect the data sequence without explicitly estimating the phase reference. A similar conclusion is reached in [32], where a suboptimal solution based on vector tracking is proposed. Nevertheless, this solution is affected by the typical problems of PLL-based receivers.

This generalized likelihood approach may be easily shown to be exactly equivalent to noncoherent sequence detection in the case of constant envelope modulations, as well as PSK in the absence of ISI [39]. Furthermore, by the approximation $\log I_{0}(x) \simeq x$ valid at high $\mathrm{SNR}$, this result may be shown to approximately hold for arbitrary modulation formats, with an excellent approximation quality in a broad range of applications [39]. Numerical results confirm that at low SNR the equivalence still holds, despite the lack of a theoretical basis. This equivalence suggests that the distinction between stochastic and deterministic time-invariant phase models might be theoretically and practically irrelevant in most cases.

\section{REFERENCES}

[1] G. Colavolpe and R. Raheli, "Noncoherent sequence detection of continuous phase modulations," this issue, pp. 1303-1307.

[2] G. Ascheid and H. Meyr, "Cycle slips in phase-locked loops: A tutorial survey," IEEE Trans. Commun., vol. COM-30, pp. 2228-2241, Oct. 1982.

[3] F. M. Gardner, "Hangup in phase-lock loops," IEEE Trans. Commun., vol. COM-25, pp. 1210-1214, Oct. 1977.

[4] F. M. Gardner, "Equivocation as a cause of PLL hangup," IEEE Trans. Commun., vol. COM-30, pp. 2242-2243, Oct. 1982.

[5] U. Mengali and A. N. D'Andrea, Synchronization Techniques for Digital Receivers. New York: Plenum, 1997.

[6] R. Raheli and G. Colavolpe, "Non-coherent sequence estimation receiver for digital modulation," assigned to Italtel S.p.A., Milan, Italy, International Patent Application n. PCT/EP98/03486, June 1998; following the Italian Patent n. MI97A 001301, June 1997.

[7] G. Colavolpe and R. Raheli, "Non-coherent sequence detection of $M$ ary PSK," in Proc. IEEE Int. Conf. Communications (ICC'97), Montreal, June 1997, pp. 21-25.

[8] G. Colavolpe and R. Raheli, "On noncoherent sequence detection of coded QAM," IEEE Commun. Lett., vol. 2, pp. 211-231, Aug. 1998. 
[9] G. Colavolpe and R. Raheli, "Noncoherent sequence detection of coded linear modulations," in Proc. IEEE Int. Conf. Univ. Personal Communications (ICUPC'98), Oct. 1998, pp. 1105-1109.

[10] J. Proakis, Digital Communications, 2nd ed. New York: McGraw-Hill, 1989.

[11] P. Y. Kam, "Maximum-likelihood carrier phase recovery for linear suppressed-carrier digital data modulations," IEEE Trans. Commun., vol. COM-34, pp. 522-527, June 1986.

[12] H. Leib and S. Pasupathy, "The phase of a vector perturbed by Gaussian noise and differentially coherent receivers," IEEE Inform. Theory, vol. 34, pp. 1491-1501, Nov. 1988.

[13] F. Edbauer, "Bit error rate of binary and quaternary DPSK signals with multiple differential feedback detection," IEEE Trans. Commun., vol. 40, pp. 457-460, Mar. 1992

[14] P. Y. Kam, S. S. Ng, and T. S. Ng, "Optimum symbol-by symbol detection of uncoded digital data over the Gaussian channel with unknown carrier phase," IEEE Trans. Commun., vol. 42, pp. 2543-2551, Aug. 1994.

[15] S. G. Wilson, J. Freebersyser, and C. Marshall, "Multi-symbol detection of $M$-DPSK," in Proc. Global Communications Conf. (GLOBECOM'89), Nov. 1989, pp. 1692-1697.

[16] D. Divsalar and M. K. Simon, "Multiple-symbol differential detection of MPSK," IEEE Trans. Commun., vol. 38, pp. 300-308, Mar. 1990.

[17] H. Leib and S. Pasupathy, "Noncoherent block demodulation of PSK," in Proc. Vehicle Technolology Conf. (VTC'90), May 1990, pp. 407-411.

[18] M. K. Simon, S. M. Hinedi, and W. C. Lindsey, Digital Communication Techniques. Englewood Cliffs, NJ: Prentice-Hall, 1995.

[19] D. Divsalar, M. K. Simon, and M. Shahshahani, "The performance of trellis-coded MDPSK with multiple symbol detection," IEEE Trans. Commun., vol. 38, pp. 1391-1403, Sept. 1990.

[20] Y. Kofman, E. Zehavi, and S. Shamai, "nd-convolutional codes-Part I: Performance analysis," IEEE Trans. Inform. Theory, vol. 43, pp. 558-575, Mar. 1997.

[21] Y. Kofman, E. Zehavi, and S. Shamai, "nd-convolutional codes-Part II: Structural analysis," IEEE Trans. Inform. Theory, vol. 43, pp. 576-589, Mar. 1997.

[22] D. Divsalar and M. K. Simon, "Maximum-likelihood differential detection of uncoded and trellis coded amplitude phase modulation over AWGN and fading channels-Metrics and performance," IEEE Trans. Commun., vol. 42, pp. 76-89, Jan. 1994.

[23] M. K. Simon and D. Divsalar, "Multiple symbol partially coherent detection of MPSK," IEEE Trans. Commun., vol. 42, pp. 430-439, Feb./Mar./Apr. 1994

[24] D. Raphaeli, "Noncoherent coded modulation," IEEE Trans. Commun., vol. 44, pp. 172-183, Feb. 1996.

[25] D. Makrakis and P. T. Mathiopoulos, "Trellis coded noncoherent QAM: A new bandwidth and power efficient scheme," in Proc. Vehicle Technology Conf. (VTC'89), May 1989, pp. 95-100.

[26] D. Makrakis and K. Feher, "Optimal noncoherent detection of PSK signals," Electron. Lett., vol. 26, pp. 398-400, Mar. 1990.

[27] M. V. Eyuboğlu and S. U. H. Qureshi, "Reduced-state sequence estimation with set partitioning and decision feedback," IEEE Trans. Commun., vol. 36, pp. 13-20, Jan. 1988.

[28] P. R. Chevillat and E. Eleftheriou, "Decoding of trellis-encoded signals in the presence of intersymbol interference and noise," IEEE Trans. Commun., vol. 37, pp. 669-676, July 1989.

[29] G. Colavolpe and R. Raheli, "Theoretical analysis and performance limits of non-coherent sequence detection of $M$-ary PSK," IEEE Trans. Inform. Theory, to be published.

[30] G. Ungerboeck, "Channel coding with multilevel/phase signals," IEEE Trans. Inform. Theory, vol. IT-28, pp. 55-67, Jan. 1982

[31] E. Biglieri, D. Divsalar, P. J. McLane, and M. K. Simon, Introduction to Trellis-Coded Modulation with Applications. New York: Macmillan, 1991.
[32] A. N. D'Andrea, U. Mengali, and G. M. Vitetta, "Approximate ML decoding of coded PSK with no explicit carrier phase reference," IEEE Trans. Commun., vol. 42, pp. 1033-1039, Feb./Mar./Apr. 1994.

[33] R. Raheli, A. Polydoros, and C. K. Tzou, "Per-survivor processing: A general approach to MLSE in uncertain environments," IEEE Trans. Commun., vol. 43, pp. 354-364, Feb./Mar./Apr. 1995.

[34] G. Ungerboeck, "Adaptive maximum-likelihood receiver for carriermodulated data-transmission systems," IEEE Trans. Commun., vol. COM-22, pp. 624-635, May 1974.

[35] G. D. Forney Jr., "Maximum-likelihood sequence estimation of digital sequences in the presence of intersymbol interference," IEEE Inform. Theory, vol. IT-18, pp. 363-378, May 1972.

[36] W. J. Weber, "Differential encoding for multiple amplitude and phase shift keying systems," IEEE Trans. Commun., vol. 26, pp. 385-391, Mar. 1978

[37] D. Raphaeli, "Decoding algorithms for noncoherent coded modulation," IEEE Trans. Commun., vol. 44, pp. 312-323, Mar. 1996.

[38] H. L. Van Trees, Detection, Estimation and Modulation Theory. New York: Wiley, 1968.

[39] G. Colavolpe, "Ricevitori a rivelazione di sequenza per trasmissioni su canali a fase incognita," Ph.D. dissertation, Univ. Parma, Parma, Italy, 1998 (in Italian). Available at: http://com.unipr.it/ people/colavolpe/giulio.html.

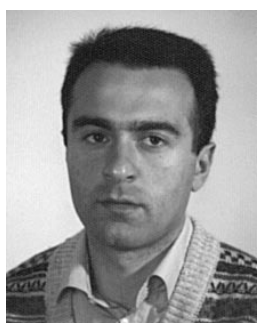

Giulio Colavolpe (S'97) was born in Cosenza, Italy, in 1969. He received the Dr.Ing. degree in telecommunications engineering (cum laude) from the University of Pisa, Italy, in 1994, and the doctoral degree in information technology from the University of Parma, Italy, in 1998.

Since 1998, he has been a Research Associate at the University of Parma. His main research interests include digital transmission theory, channel coding, and signal processing.

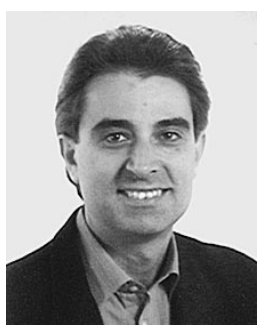

Riccardo Raheli (M'87) was born in Alezio (Lecce), Italy, in 1957. He received the Dr.Ing. degree (Laurea) from the University of Pisa, Italy, in 1983, the M.S. degree from the University of Massachusetts, Amherst, U.S.A., in 1986, and the doctoral degree (Perfezionamento) from the Scuola Superiore di Studi Universitari e di Perfezionamento, Pisa, Italy, in 1987, all in electrical engineering.

From 1986 to 1988, he was a Project Engineer with Siemens Telecomunicazioni, Milan, Italy. From 1988 to 1991, he was a Research Professor at the Scuola Superiore di Studi Universitari e di Perfezionamento S. Anna, Pisa, Italy. In 1990, he was a Visiting Assistant Professor at the University of Southern California, Los Angeles, U.S.A. Since 1991, he has been a Faculty Member at the University of Parma, Italy, where he is currently an Associate Professor of Telecommunications. His main research interests include digital transmission theory, sequence detection techniques, signal processing, and adaptive algorithms for communications. His research activity has lead to about 50 scientific publications in international journals and conference proceedings and a few industrial patents. 